\title{
The Optics of an Interferometric Gravitational-Wave Antenna
}

\author{
W. Winkler and colleagues of GEO ${ }^{1}$ \\ Max-Planck-Institut für Quantenoptik, D-8046 Garching, Germany \\ ${ }^{1}$ Names and affiliations of all authors are given at the end of the \\ paper
}

\begin{abstract}
The basic concept of an interferometric gravitational wave detector, the realization of the long light path with optical delay lines or with Fabry-Perot cavities, and the need for high light power are described. The techniques for improving the sensitivity, recycling and squeezed states of light, are considered and the consequences on the specifications of the optical components are shown. The specifications are explicitly given and particularly the influence of thermal effects is treated quantitatively.
\end{abstract}

\section{Introduction}

All realistic sources for gravitational waves that have been thought of so far are expected to provide us with extremely small signals. The most efficient emission of radiation from supernova core implosions or from coalescing binaries lasts only a few milliseconds, and for an event rate of several per month the strain in space introduced by gravitational waves (that is a change in relative distance between testparticles) may be on the order of only $h=$ $\frac{1}{2} \frac{\delta L}{L} \approx 10^{-22}$.

As described in the contribution of K. Danzmann in this issue, a Michelson interferometer is an adequate tool to look for such tiny strains. For an optimization of the sensitivity one has to optimize both - the effective light path $L$ corresponding in some sense to the interaction time between the gravitational wave and the light inside the interferometer - and the resolution for the path difference $\delta L$. With techniques available today it should be possible to reach the required sensitivity level, as we will see in the following sections. 


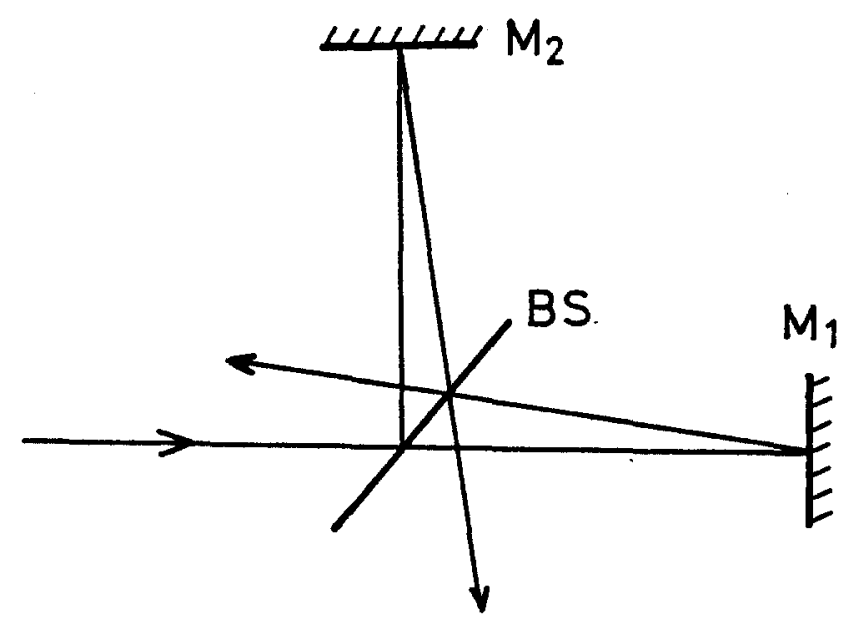

Fig. 1. Michelson interferometer with two mirrors $M_{1}$ and $M_{2}$ and a beam-splitter BS

\section{The long Light Path}

A gravitational wave passing the interferometer introduces a strain in space with opposite sign in two orthogonal directions. The path difference $\delta L$ to be measured in a Michelson interferometer is a maximum if the storage time of the light in the arms is matched to the period of the gravitational wave. For periods of milliseconds the optical light path is optimally of the order of 100 $\mathrm{km}$. In order to realize this long light path the light is sent back and forth in each arm several times before it is superposed with the other beam at the beam-splitter. The number of beams cannot be chosen very high, otherwise residual mirror motions like the thermally driven eigenmodes of the mirror substrate would limit the sensitivity of the antenna. An armlength of a few kilometers seems to be a good choice as a compromise between optimal performance of the interferometer and financial investment.

In the prototypes of gravitational wave antennas built and tested in the past, two possibilities for realizing long light paths have been investigated: optical delay lines and Fabry-Perot cavities. 


\subsection{Fabry-Perot Cavities}

In optical cavities the many beams running between the terminating mirrors are all superposed to form one intense beam [1]. The far mirror is coated for highest possible reflectivity, whereas the transmission of the near mirror determines the effective path length inside the cavity:

$$
L_{\text {eff }} \approx \frac{\ell}{T},
$$

where $\ell$ denotes the mirror separation and $T$ the power transmittance of the coupling mirror. Thus, the effective path length inside the cavity is adjustable by the proper transmittance of the input mirror. Ideally all of the light eventually goes back to the beam-splitter. Variations in path length result in a phase shift which is detected by superposition with the light from the other arm.

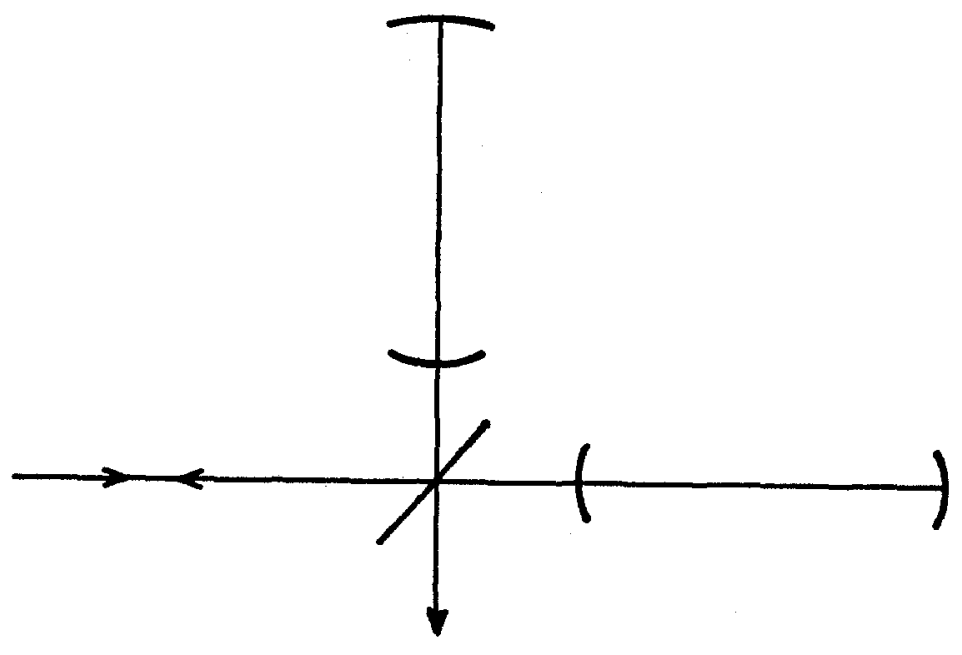

Fig. 2. Michelson interferometer with Fabry-Perot cavities

A cavity has to be designed according to the properties of the light beam, in our case a diffraction limited laser beam. Such a Gaussian beam is already determined by position and diameter $2 w_{0}$ of its focus. The beam radius $w$ is defined by the distance between the beam axis and the point where the intensity is $e^{-2}$ of its maximum value.

There is a characteristic length $b$, the so-called Rayleigh range, for each Gaussian beam, defined by

$$
b=\frac{2 \pi}{\lambda} w_{o}^{2},
$$




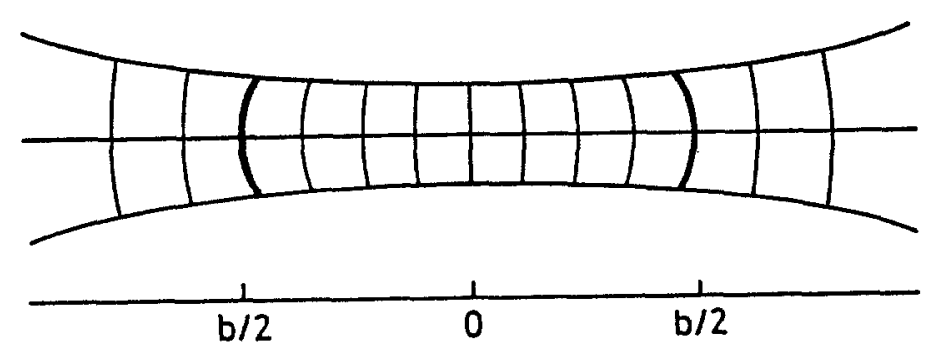

Fig. 3. Wavefronts in a diffraction limited laser beam

with $\lambda$ the wavelength of the light. The radius $w$ in the radial intensity distribution

$$
I=I_{o} \exp \left(-\frac{2 r^{2}}{w^{2}}\right)
$$

varies with distance $z$ to the focus according to

$$
w=w_{o}\left(1+\left(\frac{z}{b / 2}\right)^{2}\right)^{1 / 2}
$$

The equivalent relation for the radius of curvature $R_{w}$ of the wavefront reads as

$$
R_{w}=z\left(1+\left(\frac{b / 2}{z}\right)^{2}\right) .
$$

$b$ is the smallest radius of curvature of the wavefronts in a beam with focal diameter $2 w_{o}$, occurring at a separation of $b / 2$ from the focus.

A cavity can be formed by inserting mirrors in the light path with a curvature of the surface equal to the curvature of the wavefront. The beam is then reproduced by the subsequent reflections. A possible arrangement could be a flat mirror in the focus and a concave mirror at a distance $z$, with radius of curvature corresponding to the last equation. The average beam size is smallest in a near confocal cavity, that is a symmetric arrangement with radii of curvature close to the mirror separation:

$$
2 w=2\left(\frac{\lambda \ell}{\pi}\right)^{1 / 2}
$$

For visible light and $\mathrm{km}$-dimensions the beam size as defined above is a few $\mathrm{cm}$. In order to avoid diffraction losses, that is parts of the beam falling off the mirror edge, one has to assign a space to each reflection by a factor $S$ bigger in diameter than the beam diameter. Usually $S \approx 3$ is assumed to be 
sufficient. For more details see for instance the contribution of R.W.P. Drever in [2].

\subsection{Optical Delay Lines}

The second possibility for a multireflection scheme to realize the long light path is an optical delay line [3]. Here the subsequent reflections are more or less well separated from each other, and the light path is well defined as $L=N \ell$ with $N$ the number of beams and $\ell$ the mirror separation. The simplest case of a delay line is formed by two equally curved spherical mirrors facing each other at a separation $\ell$ equal to the radius of curvature $R$. This is the so called confocal separation, as the focal points of the two mirrors coincide.

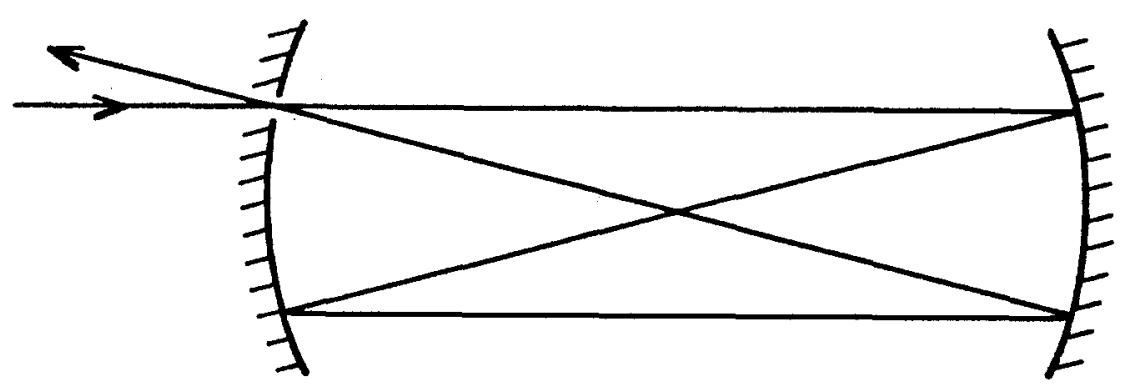

Fig. 4. Confocal delay line

The beam usually enters the delay line through a coupling hole in the near mirror. The far mirror produces an image of the coupling hole onto the near mirror symmetrically to the optical axis. (The optical axis can be defined as the line connecting the centers of curvature of the two mirrors). This image can now again be considered to represent an object, which in turn is imaged by the far mirror into the coupling hole - independent of the position of the coupling hole and the orientation of the input beam. In order to get more than four beams, the mirror separation is changed by $\Delta \ell$. As a result, the fourth beam is shifted with respect to the coupling hole by an amount proportional to $\Delta \ell$, hits the reflecting surrounding of the hole and starts a new round trip. For a particular value of $\Delta \ell$ the beam falls into the coupling hole after $N$ reflections. Usually the reflection spots are circularly arranged by a proper orientation of the input beam (see Fig. 5).

The coordinates of the reflections at the mirrors obey the following equations: 


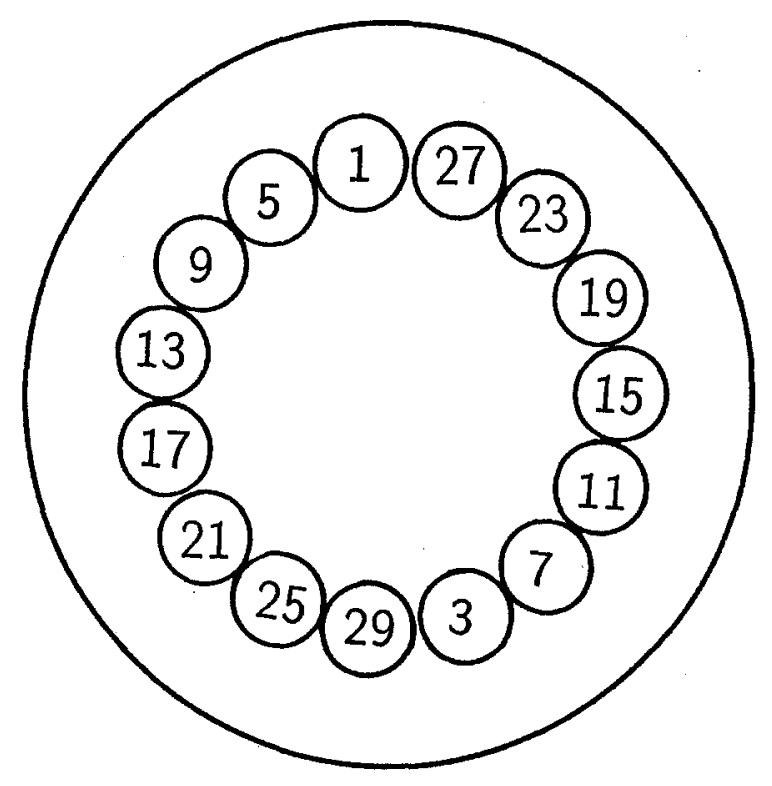

Fig. 5. Circular arrangement of reflection spots at one delay line mirror for $N=30$ beams

$$
\begin{aligned}
& x_{n}=x_{o} \cos n \Theta+\sqrt{\frac{\ell}{2 R-\ell}}\left(x_{o}+R x_{o}^{\prime}\right) \sin n \Theta \\
& y_{n}=y_{o} \cos n \Theta+\sqrt{\frac{\ell}{2 R-\ell}}\left(y_{o}+R y_{o}^{\prime}\right) \sin n \Theta
\end{aligned}
$$

$\left(x_{o}, x_{o}^{\prime}\right)$ and $\left(y_{o}, y_{o}^{\prime}\right)$ are position and slope of the input beam in $\mathrm{x}$ - and $\mathrm{y}$-direction, respectively.

In a circular arrangement the subsequent reflections seem to be rotated by $\Theta$ from one mirror to the next, whereas the reflections at one mirror are rotated by $2 \Theta . \Theta$ is defined in the usual way [3] by:

$$
\cos \Theta=1-\frac{\ell}{R} .
$$

(For the confocal mirror separation we have $\Theta=\pi / 2$ ). The mirror separation can be chosen such that after $N$ reflections the beam arrives at the same spot where it entered the delay line, in our case the coupling hole. This is the so-called reentrance condition. It is fulfilled if $N \Theta$ is equal to an integer multiple of $2 \pi$.

Delay lines have some practical advantages: position and orientation of the output beam are independent of rotations and tilts of the far mirror. 
Thus the interference quality is not spoiled by angular motions of that mirror which may be kilometers away. For practical reasons it is sometimes desirable to have a well defined optical path length - for instance to tune the storage time to a particular signal frequency, or to equalize the light paths in the two arms in order to be less susceptible to frequency fluctuations. In a delay line the optical path is $N \ell$; as the mirror separation is rigidly connected to the radii of curvature of the mirrors, it is given with the same precision as these. In a cavity the effective light path depends on the reflection losses, and these are more difficult to control.

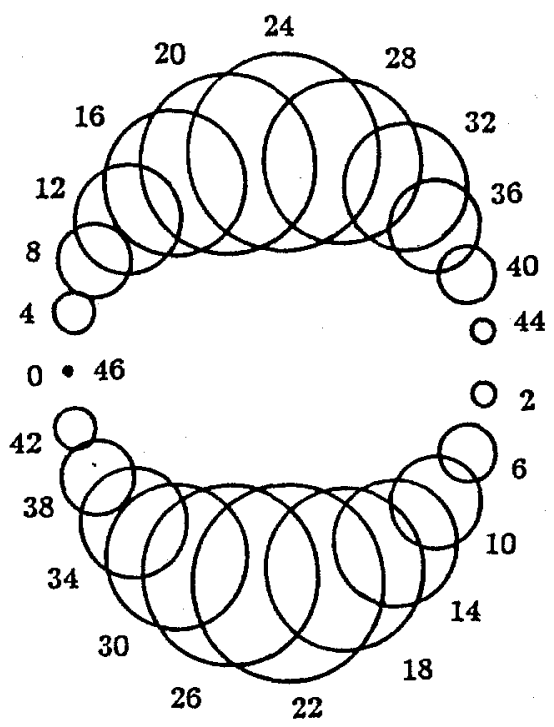

Fig. 6. Shape of the reflection spots in a delay line with minimal mirror diameter

On the other hand, there is the disadvantage of the large mirrors needed in delay lines. In a cavity the mirror diameter $D$ has to be chosen to be $D=S \cdot 2 w$ with a safety factor $S$ of at least 3 . A mirror diameter of the order of $20 \mathrm{~cm}$ is therefore sufficient for cavities with $\mathrm{km}$-dimensions and light in the near infrared.

In delay lines the different reflections have to be more or less well separated from each other, the beam has to fit through the coupling hole and no light should fall from the edges of the mirrors. For the so-called matched case where all the reflection spots have the same size, the mirror diameter would become too big, especially for a large number of beams. The mirror diameter can be minimized if the diameter of the input beam is reduced, 
allowing for a smaller coupling hole and closer packed reflections, see Fig. 6. This minimal mirror diameter $D$ is given by

$$
D=S \frac{2+\sqrt{2}}{\pi} \sqrt{\lambda L} \text {. }
$$

Thus, $D$ depends only on the wavelength of the light and the total light path. For green light, a path length of $100 \mathrm{~km}$ and a safety factor of 3 the mirror diameter would have to be about $70 \mathrm{~cm}$.

A large mirror diameter is disadvantageous because of several reasons: the thermally driven eigenresonances of the mirror substrate come close to the frequency window of interest, and it is difficult to manufacture large mirrors with the required high quality (see below). For more details see for instance the contribution about delay lines and interferometric detection of gravitational waves [4].

\subsection{Shot noise limit}

The long light path is one condition for optimized performance of an interferometric gravitational wave detector. The other one is a good resolution of changes in path difference within the frequency window of observation. Provided the influence of all noise sources can be kept small enough, the limiting noise source is the fluctuation in photo current as it is determined by the statistics of the light at the output of the interferometer. Conventional laser light at its best is described by Poisson statistics, leading to the well known $\sqrt{n}$ law: if $n$ photons are detected within a given resolution time, the uncertainty in that number is given by $\sqrt{n}$. On the other hand, a signal related to a changing path difference increases proportional to the number of photons available. Therefore, the signal-to-noise ratio improves with the square root of the number of photons, that is with the square root of the laser power. The spectral density of the mean-square fluctuation in path difference, simulated by the shot noise in an otherwise perfect interferometer, is given by

$$
S_{\delta L}(f)=\frac{\hbar c}{\pi} \frac{\lambda}{\eta P}
$$

Here $\hbar$ is Planck's constant divided by $2 \pi, c$ the speed of light, $P$ the light power at the beam splitter and $\eta$ the quantum efficiency of the photo diode. The spectral density is normalized to the bandwidth used; the units therefore are $\mathrm{m}^{2} / \mathrm{Hz}$. In order to get a linear measure, usually the square root of this quantity is given, with the unit $\mathrm{m} / \sqrt{\mathrm{Hz}}$.

Once a frequency window for observation is chosen, the relevant fluctuation in path difference can be obtained by integrating the spectral density of the noise over the frequency band in question.

The strain in space simulated by the shot noise in a perfect interferometer with an optical path length $L$, an effective light power $P$, an observational bandwidth $\Delta f$ and green light is described by 


$$
\frac{\delta L}{L}=1 \times 10^{-22}\left(\frac{50 \mathrm{~kW}}{\eta P} \frac{\Delta f}{1 \mathrm{kHz}}\right)^{1 / 2} \frac{100 \mathrm{~km}}{L} .
$$

This relation is valid for a path-length smaller than half a wavelength of the gravitational wave. A path-length of $100 \mathrm{~km}$ would be optimal for ms timescales.

Discouraging is the huge light power of $50 \mathrm{~kW}$ occuring in the last relation. It would drop to a more decent $50 \mathrm{~W}$ for the same strain sensitivity, if the frequency band of observation would be shifted from the region around $1 \mathrm{kHz}$ to around $100 \mathrm{~Hz}$, using an optical path of $1000 \mathrm{~km}$ and a bandwidth of $100 \mathrm{~Hz}$. The optimal choice of the parameters certainly depends on the characteristic timescales of the gravitational waves. Since signals occurring at a reasonable rate of about one per month are expected to have an amplitude of $10^{-21}$ at most, and since detailed information about the waveform of a gravitational wave is of basic scientific concern, one will try to improve the sensitivity as far as possible. There have been several proposals in the past, how one could proceed.

All of the concepts are attractive in themselves, and have been proven to work in principle. But in order to work properly and reliably, they pose quite stringent demands on the quality of the optical components.

\section{Techniques for improved sensitivities}

Mainly three possibilities to improve the sensitivity of an interferometer are currently under investigation: power recycling, signal recycling and the use of squeezed states of light.

\subsection{Power recycling}

As we have seen in (11), a high light power sensing the relative position of the interferometer mirrors keeps the shot noise limit of the measurement process low. It seems therefore desirable to increase the light power beyond the level provided by the illuminating laser. One possibility is to add up coherently the output power of several lasers. In order to run all the lasers at the same frequency, a small fraction of the light of a well stabilized master laser is injected into the cavities of the other lasers; these lasers oscillate at the frequency of the injected light, if it is close enough to a possible eigenfrequency $[5,6]$.

Another solution to the high power problem is to implement so-called power recycling. The idea behind it is the following: The interferometer is operated at a dark fringe at the signal output port. A fast servo loop maintains that condition, and a signal appears as a voltage or a current applied to the positioning elements. Ideally no light leaves through the signal 
output port - it leaves the interferometer through the other output port towards the laser. As seen from the laser, the interferometer acts as a mirror. By inserting another mirror $\left(M_{3}\right.$ in Fig. 7$)$ between the laser and the interferometer, a Fabry-Perot cavity is formed.

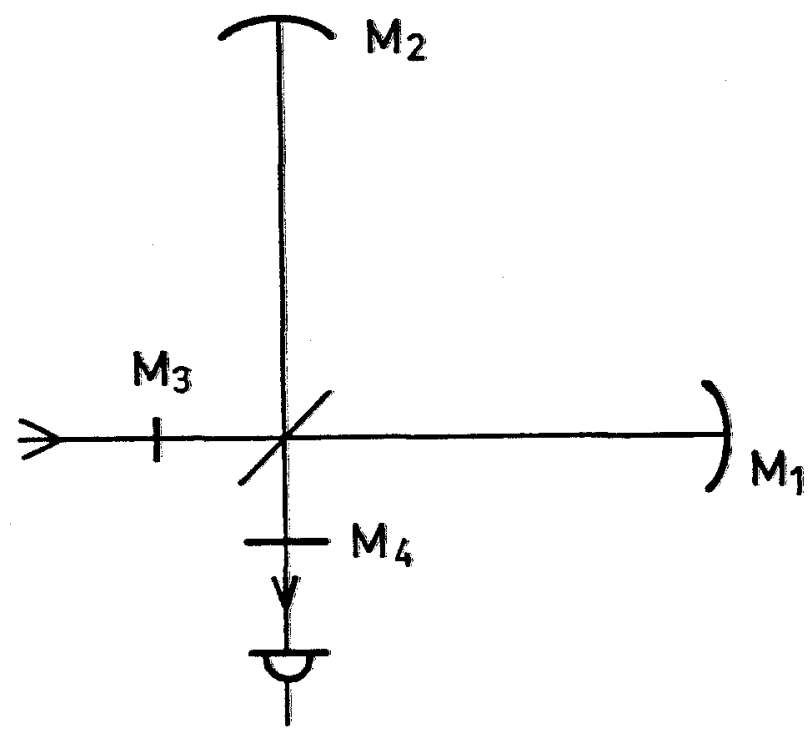

Fig. 7. Recycling the light in an interferometer: mirror $M_{3}$ for power recycling, mirror $M_{4}$ for signal recycling

The power-recycling cavity is tuned to the laser frequency, and the light power circulating inside the interferometer (and thus sensing the relative position of the mirrors) may be substantially increased if the overall power losses $\Delta P$ can be kept small. The power enhancement $G$ is given by

$$
G=\frac{P}{\Delta P}
$$

$G$ is the inverse relative power loss per total round trip inside the interferometer. Losses occur because of scattering, absorption, residual transmission of the mirrors, and poor interference. 


\subsection{Signal recycling}

A similar argument holds for the second recycling technique. As just mentioned, the interferometer is operated such that in the absence of a signal all light leaving the interferometer ideally goes back towards the laser. Analogously, light sent into the interferometer from the other side of the beam splitter leaves through the output port on that side. The interferometer therefore also acts like a mirror if one looks at it from this side of the beam splitter. By inserting a mirror $\left(\mathrm{M}_{4}\right.$ in Fig. 7 ) between interferometer and photo diode, a cavity for the signal is formed [7]. In general, this cavity will have to be tuned to a frequency different from the laser frequency: the effect of a gravitational wave is to introduce a time dependent strain in space; light being underway in a region underlying a time dependent strain in the direction of propagation can be considered as being frequency modulated - just as if the index of refraction would be modulated during the propagation through that medium. Superposition with the reference beam from the other arm can be considered as a superposition of light of the original frequency - the carrier - and of light of a frequency shifted by the frequency of the gravitational wave - the sidebands. For the carrier the interferometer is still in its condition of giving a dark fringe at the signal output port. But for the sidebands the interferometer is detuned and light is leaving towards the photodiode. Therefore the signal recycling cavity has to be tuned to the frequency of the sidebands.

The position of $\mathrm{M}_{4}$ determines the resonance frequency, and the reflectivity defines the bandwidth (together with the losses inside the interferometer, of course). In general, for a very narrow resonance it is possible to resonate one sideband only. For broader resonance it may be possible to cover both sidebands by tuning to the carrier frequency.

This technique allows to optimize the interaction time of a gravitational wave with the light, even when the light path in the interferometer arms is relatively short. It is therefore, for instance, not necessary to realize a light path of half a gravitational wavelength in each arm for optimum performance; the longer light path may equally well be established by means of signal recycling. This fact helps to overcome the problem of too large a mirror size in delay lines.

If the losses and wavefront distortions can be kept small enough, a very high recycling gain for the power and for the signal may be possible. A multipath scheme like delay lines or Fabry-Perots in the arms may no longer be necessary; the arrangement of Fig. 7 with four mirrors in total could be sufficient. A practical difficulty arises from the high light power which in this case has to be transmitted through the beam-splitter. Thermal lensing is then very likely to limit the performance of the system. For this practical reason one will therefore choose at least a few bounces in each arm before the light goes back to the beam-splitter. 
Signal recycling provides the possibility to track a signal of variable frequency, once it is detected and the subsequent shape is predictable within certain limits, like in the case of a coalescing binary. For these special sources this combines the convenience of broadband observation with the sensitivity of narrow band observation.

A very important consequence of signal recycling is the ability to regain at least part of the light that otherwise would leave the interferometer because of bad interference: this light is composed of other geometrical modes of the light-field than the fundamental one. The signal recycling cavity is tuned only to the fundamental mode; this field distribution builds up inside. The other modes are not resonant and are therefore suppressed by a factor up to the reflectivity of the signal recycling mirror. Very high order modes, originating from surface deformations with spatial wavelengths much smaller than the beam diameter, lead to diffraction losses, which certainly can not be regained.

\subsection{Squeezed states of light}

What limits the sensitivity of measuring phase differences in a Michelson interferometer - provided all other spurious signals are reduced sufficiently - is the noise due to the statistics of the light hitting the photodiode. Light of an ideal laser is described by Poisson statistics. This statistics leads to the theoretical sensitivity limit defined by (11). Surprisingly enough, it is not the fluctuations of the illuminating light that limit the performance of the system. Fluctuations of the laser light may take place either in amplitude or in phase. Fluctuations in amplitude only show up at the output of the interferometer if there is a deviation from the dark fringe at the measurement output port. A dark output stays dark, even when the illuminating light varies in amplitude.

A similar argument holds for the phase fluctuations of the laser light. In an interferometer phase fluctuations of the light give rise to a signal only if there is a path difference between the two interfering beams. For zero path difference, that is in the minimum of order zero, there is no light in the output, no matter which colour is used to illuminate the apparatus. A phase fluctuation is equivalent to a superposition of several frequencies, and thus on the zero order dark fringe no signal can be expected due to phase fluctuations in the illuminating light.

Where does the noise in the output come from when there seems to be no relation to the fluctuations of the input beam? The crucial point is the beam splitter. As Carlton Caves has pointed out, the noise due to photon statistics at the output of the interferometer can be described as originating from the zero point fluctuations of the vacuum field entering the interferometer through the normally unused input port symmetrically to the input illuminated by the laser [8] (see Fig. 8). 


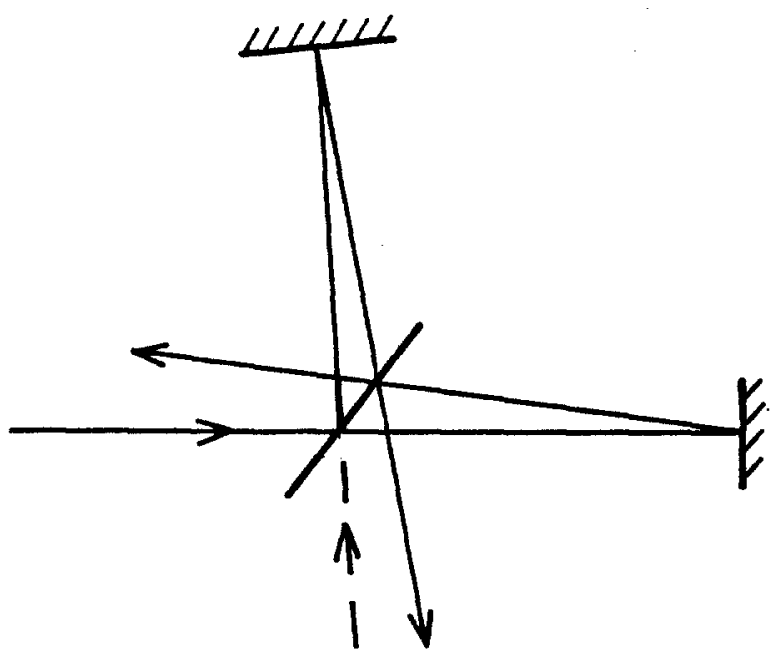

Fig. 8. Vacuum fluctuations entering the interferometer as indicated with dashed lines

These fluctuations are superposed with the light circulating inside the interferometer. The phase fluctuations are responsible for the uncertainty in the number of photons in the output, and the amplitude fluctuations give rise to the back action of the measuring process on the system via the time dependent differential light pressure on the mirrors. Caves proposed to send a particularly prepared state of the electromagnetic field into the second input port, replacing the usual zero point fluctuations, and thus reducing the fluctuations in the number of photons at the output. It is not necessary for this particular field to contain any real photons - it is sufficient to have less phase fluctuations than the vacuum field (or an ideal laser, which has the same uncertainty).

A reduction of the fluctuation in the number of photons in the output is connected with an increased fluctuation in differential light pressure onto the mirrors. But with realistic light powers and masses of several hundred kilograms this effect is negligible. Squeezed states of light have been realized in the meantime, and even the performance of an interferometer has been shown to improve by a few $\mathrm{dB}$ using squeezed states [9, 10]. Unfortunately the technique of squeezed states is not yet ready to be used in interferometric detection of gravitational waves; it will take some time to make it reliable and to overcome all the effects that tend to make the photon statistics Poissonian again.

The possible improvement of the sensitivity by use of squeezed states of light is limited by non-ideal conditions, particularly by losses, in a rather drastic way [11]. Equation (11) for a perfect interferometer can be rewritten as

$$
\delta \phi_{\min }=1 / \sqrt{n},
$$

where $n$ is the number of photons sensing the position of the mirrors within the chosen resolution time. For an ideal interferometer the gain in sensitivity 
due to the application of squeezed light is proportional to the amount of squeezing, $1-e^{-s}$. In this picture $s=0$ means no squeezing. An imperfect visibility $V$ limits the possible improvement to

$$
\delta \phi_{\min }=\left(\frac{[2(1-V)]^{1 / 2}+e^{-2 s}}{n}\right)^{1 / 2} .
$$

(The visibility is related to minimum $P_{\min }$ and maximum $P_{\max }$ output power of the interferometer via $\left.V=\left(P_{\max }-P_{\min }\right) /\left(P_{\max }+P_{\min }\right)\right)$.

Thus, even for an arbitrarily high degree of squeezing the gain in sensitivity would be limited to $[2(1-V)]^{1 / 4}$. This means that an interference minimum of $10^{-4}$, which seems to be an optimistic assumption as we will see soon, would allow a factor of 7 at most to be gained in sensitivity by utilizing perfectly squeezed light.

\section{Quality of the optical components}

There are several criteria that have to be fulfilled by the optical components. Some of them will be mentioned in the section 5.3 dealing with substrate materials. Here we will concentrate on the optical requirements. Most important is the request for low losses. Losses occur because of scattering, absorption and bad interference. We will now consider the losses introduced by a limited surface quality.

\subsection{Surface quality}

The ideal shape of the optical surfaces, especially of the mirrors, is in most of the cases either a sphere or a plane. Deviations from the ideal shape deform the wavefront of the passing beam. Depending on the spatial wavelength $\Lambda$ of these deformations, there are three regions to be considered: $\Lambda$ larger, equal to, or smaller than the beam diameter.

\subsubsection{Aberrations}

Deformations of the mirror surface on a scale larger than the beam diameter are called aberrations. They lead to a displacement and a tilt of the beams inside the interferometer. In a Fabry-Perot cavity the beam can be readjusted by properly orienting the mirrors. In a two mirror delay line not all distortions can be compensated by mirror alignment, for instance the effect of an astigmatism (that is different curvature of the mirrors in different directions). In this case the output beam in general is shifted with respect to its ideal position in radial as well as in tangential direction. Only the tangential displacement can be compensated by adjustment of the mirrors. 
The visibility at the output of the $3 \mathrm{~km}$ interferometer can be kept better than $99 \%$, if the error in slope of the mirror surface stays below $10^{-7}$ radian. If more than two mirrors are used in each interferometer arm, then position and orientation of the output beam are adjustable as well. One possibility is the use of so called retro-mirrors (see Fig. 9), where the beam leaves the delay line through a second coupling hole, hits a retro-mirror perpendicularly and retraces its original path.

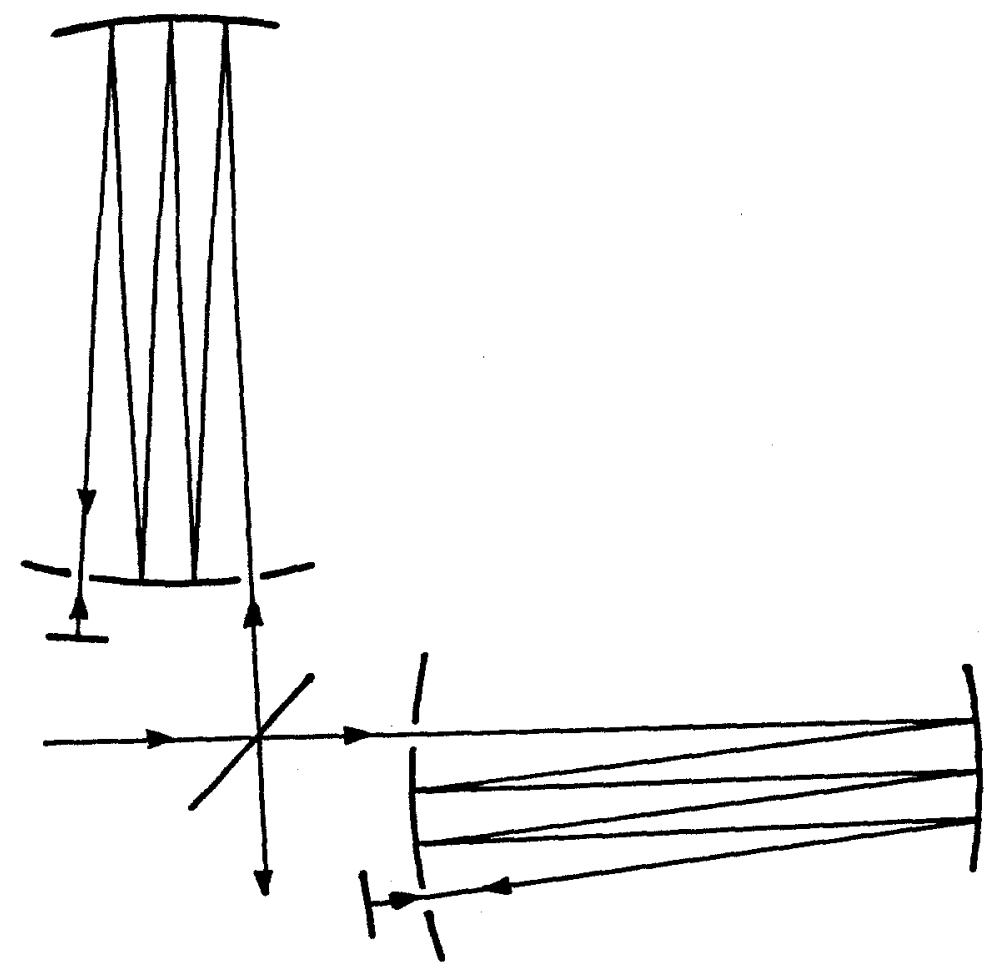

Fig. 9. Delay lines with retro-mirrors

There are several advantages of inserting a retro-mirror: for a given number of beams the number of reflection spots is smaller than in a two mirror delay line and therefore the mirrors can be kept smaller, and the recycling schemes can be realized by inserting one mirror only, without the necessity of complicated reorienting the output beam for good superposition with the input beam. 


\subsubsection{Ripple}

Surface deformations with lateral dimensions in the order of centimeters are historically called ripple. In our case the beam diameter is just of that magnitude. The main effect of a ripple on a reflecting surface is then to deform the wavefront. Computer simulations have been performed for simplified conditions [12]: all surfaces and wavefronts have been assumed to be locally spherical, and deviations in curvature have been described by a deviation $\delta s$ of the sagitta $s$ at the mirror (see also Fig. 10):

$$
s=\frac{w^{2}}{2 R}
$$

where $w$ denotes the beam radius and $R$ the radius of curvature of the wavefront. In a near confocal arrangement, where the mirror separation equals the radius of curvature, the sagitta is only a fraction of a wavelength:

$$
s \approx \frac{\lambda}{2 \pi} \text {. }
$$

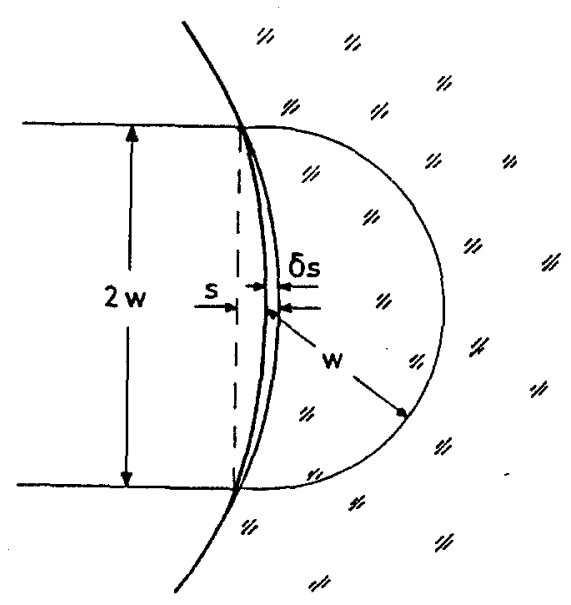

Fig. 10. A locally spherical deformation of the mirror surface and the corresponding change in sagitta

A reflection at a surface deformed by $\delta s$ spoils the relative minimum at the output of an otherwise perfect interferometer to

$$
\frac{P_{\min }}{P} \approx 10^{-3}\left(\frac{\delta s}{\lambda / 100}\right)^{2}
$$


In a real setup there are deformations at each reflection. If we assume a statistical deformation of the relevant surfaces with a standard deviation $s_{d}$ of the Gaussian - distributed surface amplitudes, then after $N$ reflections the relative minimum at the output deteriorates to

$$
\frac{P_{\min }}{P} \approx 5 \times 10^{-2} \frac{N}{34}\left(\frac{s_{d}}{\lambda / 100}\right)^{2} .
$$

This relation tells us that the wavefront deformation produced by reflections at a statistically deformed surface on the average grows proportional to the square root of the number of reflections $N$, and the minimum deteriorates with $N$. If we assume 34 reflections, then the deviations of the optical components from their ideal shape on a scale in the order of the beam size has to stay below $\lambda / 200$ in order to allow a power enhancement by a factor of 100 via power recycling. This is a fairly hard condition, as the beam size in the final detectors will be somewhere between 5 and $10 \mathrm{~cm}$ (depending on the particular layout), and the amplitudes of the surface irregularities usually grow with the spatial wavelengths considered. Today's state of the art is very close to these goals. Deviations from an ideal shape for an aperture of $250 \mathrm{~mm}$ have been measured with a lateral resolution of $1 / 2 \mathrm{~mm}$ and an accuracy of a few Angstroms [13].

The demands on the surface quality at scales comparable to the beam size can be somewhat reduced if the technique of signal recycling is implemented (see the remarks in section 3.2 on signal recycling). Most of the light power leaving the interferometer through the output port is due to bad interference. It hits the signal recycling mirror and is sent back into the interferometer. But only the fundamental mode is resonant; higher modes are suppressed corresponding to the reflectivity of the signal recycling mirror.

\subsubsection{Micro-roughness}

Surface deformations with spatial wavelengths $\Lambda$ smaller than a few $\mathrm{mm}$ - that is the order of magnitude of the beam size for small optical setups - are named micro-roughness in the usual terminology. In the large scale interferometers the beam size comes close to $10 \mathrm{~cm}$, and therefore we have to include surface deformations up to spatial wavelengths of a few $\mathrm{cm}$. Microroughness is one of the most important reasons for light scattering, and scattering is mostly the dominant loss mechanism in optical experiments. For a quantitative description of the scattering losses the quantity TIS (total integrated scattering) is used. It gives the fraction of light that leaves the main beam by propagating into arbitrary directions:

$$
T I S=\frac{\delta P}{P}=\left(4 \pi \frac{\delta s_{\mathrm{rms}}}{\lambda}\right)^{2}
$$


It is exactly the microroughness that defines the relevant surface deformation in (20). If we require the scattering losses to be small enough to allow a recycling gain of a factor of 100 in an interferometer with 34 reflections, then the last equation tells us that $\delta s_{\text {rms }}$ has to be kept below $\lambda / 730$, that is better than a nanometer for visible light. Within the last few years there has been an impressive progress in manufacturing high quality optical components. The rms-value $\delta s_{\text {rms }}$ of the surface deformations for $\Lambda$ smaller than a few $\mathrm{mm}$ can now be kept well below one tenth of a nanometer. It is again a hard (but manageable) condition, to extend the range of spatial wavelengths up to the required few $\mathrm{cm}$.

\subsection{Coating}

In order to minimize reflection losses, the optical components are coated with dielectric layers. The art of producing high quality coatings has been brought to a very high standard within the last few years. Mirrors for visible light are available with overall reflection losses of less than $10 \mathrm{ppm}$ (parts per million), and an absorption of hardly measurable $2 \mathrm{ppm}$. For infrared light, mirrors with even better performance have been made.

Some development work is still left to be done with respect to the size of the components involved. The very high quality mirrors made so far have a size of a few $\mathrm{cm}$ only, whereas for an interferometric gravitational wave antenna components with a size of several decimeters will be needed. Again, just as with the grinding procedure, it is more difficult to get extremely smooth surfaces up to spatial wavelengths in the decimeter region. As an example in Fig. 11 a scanline across a mirror used in the Garching prototype is shown, before and after coating, respectively.

No attempt has been made to produce a coating with constant thickness across the whole mirror, since only a circular area is used for the reflection spots. The coating therefore was done by sputtering from an off-axis ion source, while the mirror was rotated about its symmetry axis. As one can see from the pictures, the shape of the same surface (measured as deviation from an ideal sphere) looks totally different for the coated and for the uncoated case on a scale of several tens of nanometers. This is not surprising, since the overall thickness of the coating is about 5 micrometers, and a variation of 50 nanometers corresponds to a relative change of $1 \%$ only.

\subsection{Density fluctuations}

Wavefront deformations do not only occur when the beam is reflected at a non-ideal surface, but also when it is transmitted through a not perfectly homogeneous material, one that shows a gradient of the index of refraction across the light path. These variations result from density fluctuations, inhomogeneous distribution of the components composing the substrate, or from 


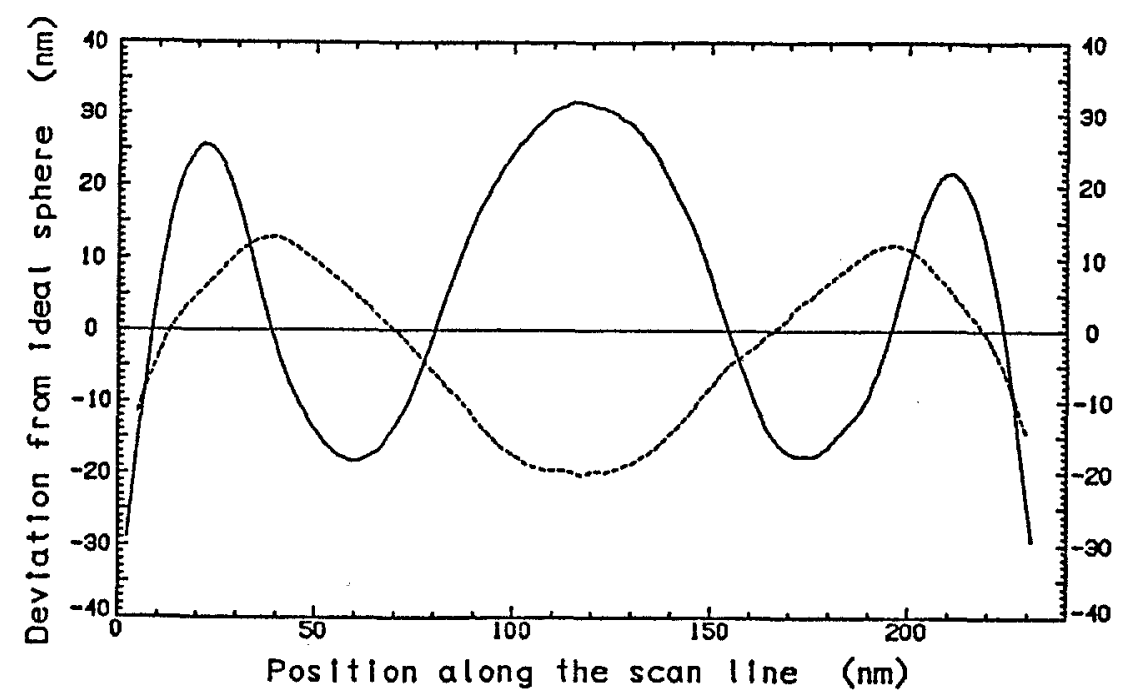

Fig. 11. Scanlines across a mirror; dashed line: before and solid line: after coating

temperature gradients (see the following section). In inhomogeneous materials different parts of the wavefront will see different optical path-lengths, leading to a deformation of the wavefront. Fluctuations of the index of refraction therefore have the same effect on the wavefront as a deformed mirror surface.

Just as in the case of surface deformations, the amplitudes of the fluctuations in the index of refraction grow with growing spatial wavelengths. The specifications for large scale interferometers are therefore harder to meet also in this respect. To give an example: The most homogeneous fused silica (a commonly used material for high quality optics) shows fluctuations of the index of refraction by $5 \times 10^{-7}$ on a scale on the order of decimeters, that is just the beam diameter in the large interferometers. The path difference between the beam axis and outer parts of the beam, as inferred by these inhomogeneities, may then be up to $5 \times 10^{-8} \mathrm{~m}$ for a $10 \mathrm{~cm}$ thick component, that is $\lambda / 10$ for green light. Such a wavefront deformation would drastically limit the possible power enhancement by power recycling (see for instance (18)). The difference in light path that different parts of the wavefront see on their way through the interferometer could at least partly be compensated for by properly shaped surfaces of the components or compensation plates. (It is planned to include this topic in a research and development contract with Zeiss, Oberkochen). In addition, as already mentioned, the 
implementation of signal recycling also reduces the losses by bad interference. This statement holds in particular for the lowest order modes, that are preferrably produced by the density fluctuations, and that are most effectively suppressed by signal recycling. But in any case the homogeneity of the substrates for the large antennas will have to be extremely high.

\section{Thermal effects}

Absorption at the components also contributes to the losses inside the interferometer. It is not the loss of light that worries us. To allow a power recycling gain of 100 , the relative loss at each of the assumed 34 reflections would have to stay below $300 \mathrm{ppm}$ only. This is no problem, since an absorption level in the ppm region is already possible. More severe are the thermal effects produced by the absorbed light power: thermal expansion and thermal lensing.

\subsection{Thermal expansion}

Let us consider a reflection at a mirror with an absorbing coating. For all relevant materials the heat will be removed by conduction rather than by radiation. The temperature profile and the related deformation by thermal expansion have been calculated [14], but the formalism and the results are somewhat complicated to handle. A good estimate of the effects in question can be obtained by the following consideration [12]: in a Gaussian beam most of the power is contained inside a circular area with radius $w$ around the beam axis. Most of the heat produced at the reflection spot is therefore also limited to that area. The heat is removed by thermal conduction into the substrate. The steepest temperature gradient occurs in the hemisphere inside the substrate with its center at the beam axis, and its radius equal to the beam radius. Eventually the whole substrate is heated (by a small amount), and radiates the power away. In the equation for the heat conduction the relevant quantities are: the absorbed light power $P_{a}$, the heat conductivity $\kappa$, the temperature drop $\delta T$ across the hemisphere, the area $A=2 \pi w^{2}$ through which the heat is transported, and finally $\alpha$, the coefficient of thermal expansion.

$$
P_{\mathrm{a}}=\kappa A \nabla T \approx \kappa 2 \pi w^{2} \frac{\delta T}{w},
$$

The temperature drop $\delta T$ causes the hemisphere to expand by

$$
\delta s \approx \alpha w \delta T / 2 .
$$

A combination of the last two equations gives 


$$
\delta s \approx \frac{\alpha}{4 \pi \kappa} P_{\mathrm{a}} .
$$

This equation tells us that the thermal deformation $\delta s$ does not depend on the beam size - it is the same for small and for large scale interferometers. On the other hand, the sagitta $s$ is close to $\lambda / 2 \pi$ for all cases (see Equations (16) and (17)). Therefore, heating effects are of same magnitude for large and for small interferometers.

In order to minimize the influence of thermal deformation onto the performance of the interferometer, the absorption has to be kept very small. The high quality coatings available today show an absorption in the ppm range for visible and for infrared light. In addition one has to use substrate materials with a small ratio between expansion coefficient and thermal conductivity. For a description of the effect of such a deformation on the performance of the interferometer we can tuse the relations described under the heading $R$ ipple of the last section. To give an example: $600 \mathrm{~mW}$ of absorbed light power at one reffection spot on a frused sillica substrate degrades a perfect interference minamum to $1 \%$, allowing a power recycling gain of less than 100 only.

\subsection{Thermal lensing}

The wavefront of a beam passing through a locally heated substrate is possibly deformed because of the temperature dependence $\beta=\partial \mathrm{n} / \partial \mathrm{T}$ of the index of refraction. A temperature gradient may occur because of absorption in the coating, or absorption along the light path in the substrate. The optical path along the beam axis differs from the optical path of outer parts of the beam by

$$
\delta s \approx \frac{\beta}{4 \pi \kappa} P_{a}
$$

$P_{a}$ is the light power absorbed either in the coating or inside the substrate. Thermal lensing puts more stringent limitations on the tolerable level of absorbed light power than thermal expansion, since for all relevant materials $\beta$ is larger than $\alpha$. Again the example of fused silica: $20 \mathrm{~mW}$ of absorbed light power are sufficient to deteriorate a perfect minumum to $1 \%$ by thermal lensing. For this reason all substrate materials through which the light is transmitted have to be extremely pure and homogeneous in order to keep the absorption small.

The low absorption levels reached today can no longer be measured with calorimeteric techniques. More sensitive measurements have been proposed by Olmstead et al. [15] and Boccara et al. [16]. They are based on the effects of thermal expansion and thermal lensing. A strong laser with the wavelength in question is sent to the component to be investigated. The change in orientation of a weak and narrow probe beam, reflected at or transmitted through the locally heated substrate, and scanned across the 
heated area, is monitored with a position sensitive photodiode. Absorbed light powers on the order of $10^{-7}$ watts are detectable. The Orsay and the Garching groups have used this technique to test their optical components $[17,18]$.

\subsection{Substrate materials}

The substrate materials have to meet several conditions. They have to be stable in shape, even on a scale of about one hundredth of a wavelength over a scale comparable to the beam diameter. Next, the mechanical quality factor has to be high, that is the mechanical internal damping has to be small in order to concentrate the thermally driven motions to a narrow bandwidth around the resonance frequencies. The resonances in turn have to be kept well outside of the frequency window of observation. In this way the tails of the resonances extending to the frequencies of observation can be kept small enough. This topic is covered in more detail in the contribution of A. Rüdiger in this issue.

The optical components should not be magnetic, even magnetic impurities have to be avoided, in order to exclude motions introduced by fluctuating electromagnetic fields.

As stated in the last sections, for minimum thermal deformation the ratio between the coefficients of thermal expansion and thermal conductivity has to be small. If the beam is transmitted through the substrate, then for minimum thermal lensing the absorption inside the material and the ratio between temperature dependence of the index of refraction and thermal conductivity has to be small.

There are several materials which meet the requirements.

Table 1.

\begin{tabular}{|c|c|c|}
\hline material & $\alpha / \kappa\left(10^{-8} \mathrm{~m} / \mathrm{W}\right)$ & $\beta / \kappa\left(10^{-8} \mathrm{~m} / \mathrm{W}\right)$ \\
\hline fused silica & 33 & 1000 \\
\hline ULE & \pm 2.3 & 850 \\
\hline silicon & 1.28 & - \\
\hline sapphire & 28 & 60 \\
\hline diamond & 0.13 & 1 \\
\hline
\end{tabular}

First of all, there are the materials normally used for optical applications, like fused silica, ULE and Zerodur. Unfortunately Zerodur, a special glass ceramic made for very low thermal expansion, is ruled out because of its high mechanical damping. Much better in this respect are ULE (also a material with low thermal expansion) and fused silica. They are possible candidates for mirror substrates. Despite their fairly strong thermal lensing, the very 
low absorbing versions may be used for the beam-splitter or even for the coupling mirror of a cavity, as long as the light power is not too high.

Components at which the light is only reflected and not transmitted, like delay-line mirrors or end mirrors of cavities, are allowed to be made from opaque materials. Silicon would be a very good choice: it has a high mechanical quality factor, is very unsusceptible to thermal deformation and can be made in large pieces.

Sapphire also has a high quality factor and shows less thermal lensing than fused silica. But so far it cannot be made in large and very homogeneous pieces. There are also problems related to its birefringence.

Beryllium oxide would also be well suited, but it is not listed here because of the toxic dust produced during the grinding procedure.

The ideal substrate would be diamond because of its extraordinarily high thermal conductivity. At present it is certainly unrealistic to count on that material. But slices of polycrystalline diamond with decimeter diameter and fractions of a millimeter thick can already be made. So it is not totally impossible that at some time sufficiently large pieces of artificial diamond will be available.

\section{Performance limitations due to thermal effects}

During the last few years an impressive progress in manufacturing high quality optics took place. It is therefore sound to assume that the optical components of an interferometer can be produced with the required specifications to get an appropriate interference quality. But, after all, the wavefronts may eventually be deformed by thermal effects. In order to quantify their influence on the performance of an interferometer, computer simulations based on the simplified models described above have been carried out [12]. An interferometer with power recycling has been assumed. The implementation of signal recycling would improve (but also complicate) the situation somewhat. The order of magnitude of the effects becomes already clear in the plots of Figs. 12 to 14 . Here the input power to be delivered by the laser is plotted against the power circulating in an interferometer with power recycling. Figure 12 relates to an interferometer with a Fabry-Perot cavity in each arm, and Fig. 13 describes a system with the same parameters, but with delay lines in the arms. In both cases Rayleigh scattering of $200 \mathrm{ppm}$ was assumed (as occurring in $10 \mathrm{~cm}$ of fused silica and green light), 20 ppm absorption inside components traversed by the light, 34 reflections in each arm (or an equivalent finesse in the case of Fabry-Perots), $30 \mathrm{ppm}$ total loss and $5 \mathrm{ppm}$ absorption in the coating. The losses were assumed to vary between the different components or between the different reflections on the average by $10 \%$. 


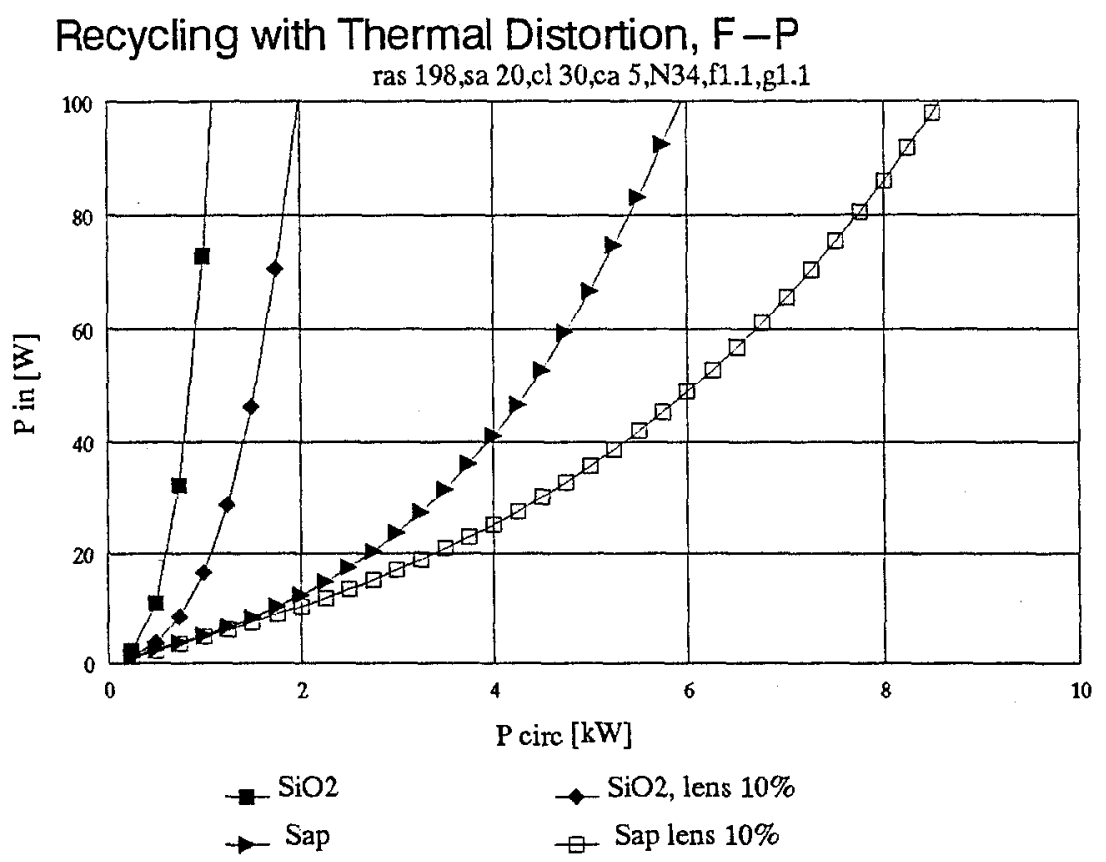

Fig. 12. Required input power $P_{i n}$ as a function of the circulating power $P_{\text {circ }}$ in a thermally distorted interferometer containing Fabry-Perots in the arms.

filled squares: all components made from fused silica

filled diamonds: the same, but the thermal lens in the beam-splitter is compensated by a compensation plate down to $10 \%$

filled triangles: all components made from sapphire

empty squares: the same, but compensation of the thermal lens in the beamsplitter

The most critical point for Fabry-Perot systems (Fig. 12) is the thermal lens in the coupling mirror, produced by absorption in the coating which is exposed to the enhanced power inside the cavity. This explains the striking improvement from the curve with filled diamonds to that with filled triangles, where the thermal lens in the substrate is reduced by using sapphire. The thermal lens in the beam-splitter is of minor importance, as its reduction down to $10 \%$ by use of a compensation plate only leads from the curve with filled squares to that with filled diamonds.

Delay line systems (Fig. 13) are less susceptible to thermal deformations than those with Fabry-Perots. Here the dominating effect is the thermal lens in the beam-splitter; its successively better compensation gives a correspondingly better performance, as shown in the figure. Starting with a fused silica beam-splitter (filled squares), a compensation of the thermal lens down to $10 \%$ (filled diamonds), a sapphire beam-splitter (filled triangles) and finally a compensated sapphire beam-splitter (empty squares) have 


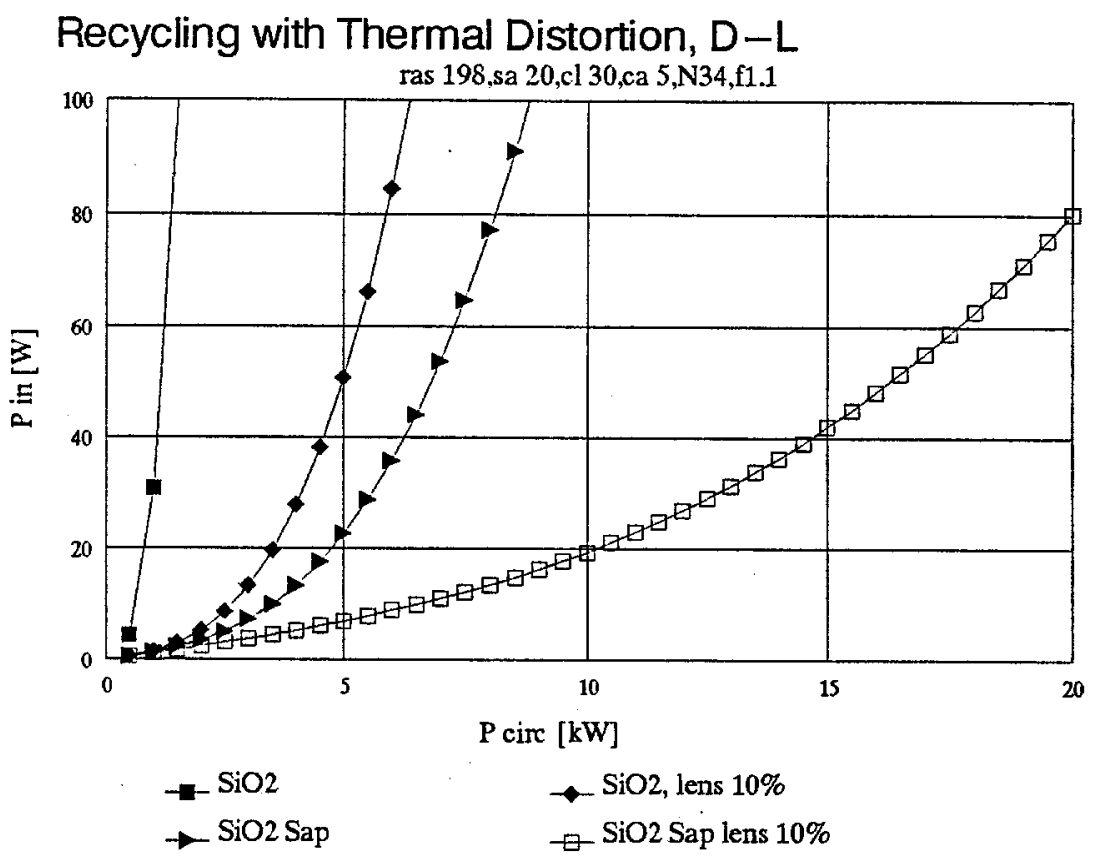

Fig. 13. Required input power $P_{i n}$ as a function of the circulating power $P_{\text {circ }}$ in a thermally distorted interferometer containing delay-lines.

filled squares: all components made from fused silica

filled diamonds: the same, but the thermal lens in the beam-splitter is compensated by a compensation plate down to $10 \%$

filled triangles: fused silica mirrors and sapphire beam-splitter

empty squares: the same, but compensation of the thermal lens in the beamsplitter

been assumed. The thermal expansion of the mirrors is of minor importance, as for all four lines mirrors made from fused silica have been assumed.

Finally in Fig. 14 some optimistic assumptions have been made as far as the materials are concerned. But they show that even light powers of $100 \mathrm{~kW}$ at the beam-splitter are conceivable. The common parameters for all four lines are: Rayleigh scattering $200 \mathrm{ppm}$, absorption in the substrate $10 \mathrm{ppm}$, coating loss $10 \mathrm{ppm}$, coating absorption $1 \mathrm{ppm}, 34$ beams, a variation of the losses of $10 \%$, and a compensation of the thermal lens in the beam-splitter down to $10 \%$.

It should be mentioned that Figs. 12 to 14 are based on green light. For infrared light the situation is still more promising, since the losses (scattering and absorption) usually are lower there. 


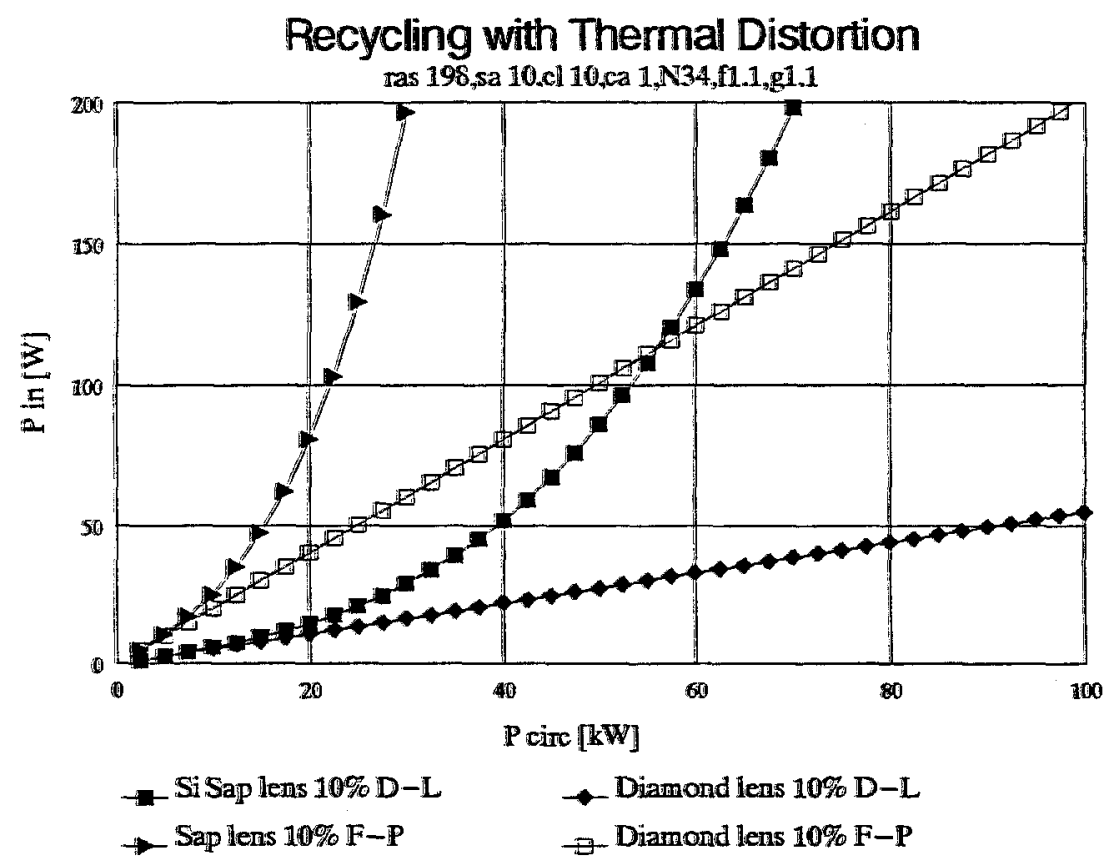

Fig. 14. Required input power $P_{\text {in }}$ as a function of the circulating power $P_{\text {circ }}$ in a thermally distorted interferometer.

filled triangles: a Fabry-Perot system made of sapphire

filled squares: a delay-line system with silicon mirrors and sapphire beam-splitter empty squares: a Fabry-Perot system made of diamond

filled diamonds: a delay-line system made of diamond

\section{Stabilization of the light}

For completeness one should also mention the optics that is needed to stabilize the laser light in frequency and in its geometrical properties, before it is sent into the interferometer. The necessity for these kinds of stabilization arises becanse fluctanations in the beam parameters produce spurious signals in connection with small, but practically unavoidable asymmetries inside the interferometer.

\subsection{Frequency stabilization}

One example of such a mechanism is the signals produced by frequency fluctuations in connection with a path difference $\Delta L$ between the interfering beams. The fluctuations $\delta \phi$ in phase difference as simulated by the frequency noise $\delta y$ of the light are given by

$$
\delta=2 \pi \frac{\Delta L}{c} \delta \nu .
$$


The path difference $\Delta L$ depends on the level of symmetry between the two arms. As described above in Sect. 2.2, the path length $L$ in a delay line is rigidly related to the radius of curvature of the mirrors. With existing technology one can hope to get the radius of curvature of the $3 \mathrm{~km}$ mirrors equal to about $10^{-3}$. So with 30 beams one has therefore to expect a path difference of about $100 \mathrm{~m}$. This path difference can in principle be made to disappear if retro-mirrors are used. But there is another effect which poses demands on the frequency stability in the same order of magnitude as the path difference between the main beams [4]: scattered light with a huge path difference with respect to the main beam and eventually interfering with it also leads to spurious signals.

A small part of the laser light is therefore sent to a stable Fabry-Perot cavity. The laser frequency is servoed to maintain resonance of the light in that cavity. Stable means in this context that in the frequency window of observation of the interferometer the cavity mirrors are allowed to show very small motions only. Even thermally driven motions at eigenfrequencies are too large. The frequency servo would then make the frequency follow these motions. The cavity is therefore either made rigid enough to have all eigenfrequencies above the frequency window in question, or uses suspended mirrors just like the interferometer. In a second stage the frequency is stabilized with respect to the light path of the interferometer itself - the quietest reference we have. In practice this can be done by stabilizing the laser frequency to the length of the power recycling cavity.

\subsection{Mode cleaner}

Another mechanism for spurious signals to appear because of unstabilities of a real laser beam are fluctuations in the beam geometry combined with proper asymmetries between the interferometer arms. A simple example is a lateral displacement $\delta y$ of the beam combined with an angular misalignment $\alpha$ of the beam-splitter. The path-difference simulated in this case is

$$
\delta L=4 \alpha \delta y .
$$

To give a numerical example: at the relevant frequencies the beam of an Argon ion laser shows fluctuations in beam position of about $10^{-10} \mathrm{~m} / \sqrt{\mathrm{H}} \mathrm{z}$. This would require the angle of misalignment to stay below $10^{-9}$ radian certainly not easy to fulfil, even for a servo system to maintain the alignment.

Similar relations hold for other kinds of beam fluctuations, for instance a pulsation in beam width combined with differently curved wavefronts of the interfering beams. Spurious signals of that sort can be suppressed by inserting a so called mode cleaner between the laser and the interferometer [19]. This mode cleaner is essentially a non-confocal Fabry-Perot cavity, where the mirror separation is chosen to resonate only one eigenmode of the 
electromagnetic field. Any fluctuation in beam geometry can be described as admixtures of other modes, which are not resonant in the cavity - they are not transmitted, they are reflected. The transmitted beam in consequence fluctuates in power - but power fluctuations are made ineffective in the signal output anyhow by use of a nulling method.

Besides mode cleaners, single mode fibers have also been used in the prototype experiments to suppress geometrical beam fluctuations. In addition, it is easier to handle position and orientation of the beam when fibers are used. But so far no fibers have been produced to stand more than one Watt of transmitted single-mode light power. For higher laser power it will therefore be necessary to use mode cleaners again.

\section{Conclusion}

The specifications for the optical components in a large baseline interferometric gravitational wave detector are at the limits of the well advanced technology today: surfaces smooth to better than $10^{-10} \mathrm{~m}$ on a scale of up to a few centimeters; on a larger scale the deviations of the surface shape with respect to an ideal sphere have to stay in the nanometer region. The losses, particularly the absorption, are tolerable only at a level of a few ppm. The beam-splitter, and particularly the coupling mirror of a Fabry-Perot, have to be made of extremely homogeneous and pure materials. Tests and first results have shown that the problems are manageable.

\section{List of Authors}

W. Winkler, J. Chen, K. Danzmann, P.G. Nelson, T.M. Niebauer, A. Rüdiger, R. Schilling, K.A. Strain, L. Schnupp, H. Walther, Max-Planck-Institut für Quantenoptik, D-8046 Garching, Germany; J. Hough, A.M. Campbell, C.A. Cantley, J.E. Logan, B.J. Meers, E. Morrison, G.P. Newton, D.I. Robertson, N.A. Robertson, S. Rowan, K.D. Skeldon, P.J. Veitch, H. Ward, Department of Physics, University of Glasgow, Glasgow, UK; H. Welling, P. Aufmuth, I. Kröpke, D. Ristau, Laser-Zentrum and Institut für Quantenoptik, Universität Hannover, D-3000 Hannover, Germany; J.E. Hall, J.R.J. Bennett, I.F. Corbett, B.W.H. Edwards, R.J. Elsey, R.J.S. Greenhalgh, Rutherford Appleton Laboratory, Chilton, Didcot, UK; B.F. Schutz, D. Nicholson, J.R. Shuttleworth, Department of Physics, University of Wales, Cardiff, UK; J. Ehlers, P. Kafka, G. Schäfer, MaxPlanck-Institut für Astrophysik, D-8046 Garching, Germany; H. Braun, Bauabteilung der Max-Planck-Gesellschaft, D-8000 München, Germany; V. Kose, Physikalisch-Technische Bundesanstalt, D-3300 Braunschweig and D-1000 Berlin, Germany. 


\section{References}

1. A. E. Siegmann, Lasers (University Science Books, Mill Valley, 1986).

2. The Detection of Gravitational Radiation, edited by D. Blair (Cambridge University Press, 1991).

3. D. Herriot, H. Kogelnik, and R. Kompfner, Appl. Opt. 3, 523 (1964).

4. W. Winkler, in The Detection of Gravitational Radiation, edited by D. Blair (Cambridge University Press, 1991).

5. C.N. Man, A. Brillet, Lecture Notes in Physics 212, 222 (1984).

6. C.N. Man, A. Brillet, Opt. Lett. 9, 333 (1984).

7. B. J. Meers, Phys. Rev. D 38, 2317 (1988).

8. C. M. Caves, Phys. Rev. D 23, 1693 (1981).

9. M. Xiao, L. A. Wu, and H. J. Kimble, Phys. Rev. Lett. 59, 278 (1987).

10. P. Grangier, R. E. Slusher, B. Yurke, and A. La Porta, Phys. Rev. Lett. 59, 2153 (1987).

11. J. Gea-Banacloche and G. Leuchs, J. Mod. Opt. 36, 1277 (1989).

12. W. Winkler, K. Danzmann, A. Rüdiger, and R. Schilling, Phys. Rev. A, 44, 7022 (1991).

13. K. Freischlad, M. Küchel, W. Wiedmann, W. Kaiser and M. Mayer, Proc. SPIE 1332, 8, (1990).

14. P. Hello and J. Y. Vinet, J. Phys. (France) 51, 2243 (1990).

15. M. A. Olmstead, N. M. Amer, and S. Kohn, Appl. Phys. A 32, 141 (1983).

16. A. C. Boccara, D. Fournier, W. Jackson, and N. M. Amer, Opt. Lett. 5, 377 (1980).

17. N. Man, private communication

18. M. Engl, Diplomarbeit, (Garching, 1991), unpublished.

19. A. Rüdiger, R. Schilling, L. Schnupp, W. Winkler, H. Billing, and K. Maischberger, Optica Acta 28, 641 (1981). 